\title{
Comparison of modelling and measurement results of spectra emitted by a programmable lamp
}

\author{
M. Feldzensztajn, ${ }^{* 1,2}$ A. Mazikowski ${ }^{1}$ \\ ${ }^{1}$ Department of Metrology and Optoelectronics, Faculty of Electronics, Telecommunications and Informatics, \\ Gdańsk University of Technology, Narutowicza Street 11/12, 80-233 Gdańsk, Poland \\ ${ }^{2}$ NIVISS Sp. z o.o. Sp. k., Rdestowa Street 53D, 81-577 Gdynia, Poland
}

Received June 12, 2018; accepted June 20, 2018; published June 30, 2018

\begin{abstract}
Nowadays artificial light with an optimized spectrum is used in many different applications. For instance, it is widely used in museums and exhibitions to illuminate the art. Moreover, such light stimulates the growth of plants, or can be applied in industry and healthcare. To achieve the best results for each application, the adjusting of a dedicated spectrum is required, which is a complicated task. The ongoing research is focused on simplifying this process. In this paper we present the developed lamp with a programmable spectrum, and its user interface. Predicted and measured output spectra are investigated.
\end{abstract}

During the last years there have been profound changes in lighting industry. Traditional incandescent bulbs were replaced by halogen and compact fluorescent lamps [1]. Recently, LEDs and LED based sources became the most efficient light sources, with a high value of color rendering index [2]. Moreover, a growing number of applications have emerged that require illumination with the spectra tailored to their individual needs, rather than ordinary white light.

The light of a dedicated spectrum is often used in museums, horticulture, industry or healthcare [3-6]. Due to a large number of possible applications, the light source with a fixed spectral characteristic is not very effective. Thus, to make a step forward, scientists and engineers are working on the development of a lamp which makes it possible for the user to freely form the light spectrum, allowing the interesting features of an illuminated object to be enhanced.

Such a lamp was also developed at the Department of Metrology and Optoelectronics of the Gdańsk University of Technology. This device is based on an integrating sphere, dedicated diffusive coating and several narrowband and wideband LEDs. The LED engine is mounted inside the integrating sphere. The device is also equipped with a controller with an intuitive graphical user interface operated by a touch screen.

Modifying a dedicated spectrum might be a complicated task. To make it easier, the spectrum is calculated, based on user's input, and displayed on the controller's screen.
In this paper we focused on the comparison of the calculated and the actual spectra emitted by the lamp, which allows for estimation of calculated spectrum accuracy [7].

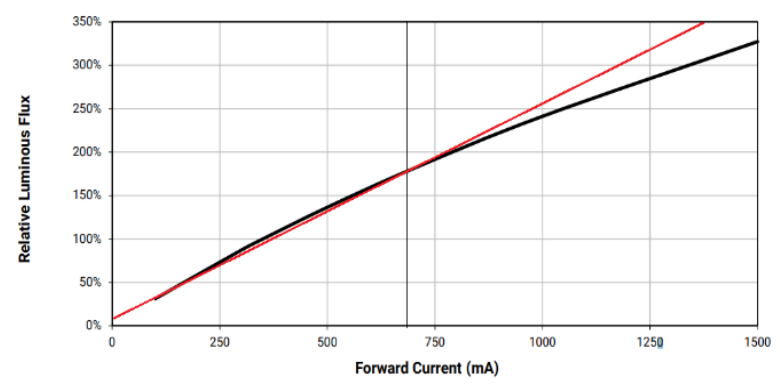

Fig. 1. Optical performance of white $\mathrm{LED}\left(\mathrm{TJ}=85^{\circ} \mathrm{C}\right)$ and its linear estimation [7].

In our programmable lamp, LEDs are driven by $650 \mathrm{~mA}$. In this current range the characteristics of LEDs used in this device are quasi-linear, as shown in Fig. 1. The influence of spectral characteristics of the integrating sphere is included in the calculation of the expected theoretical spectrum, because of the measurements each channel includes this factor. The spectrum of each LED channel of the lamp used in the calculations was measured in a stable state to minimize the influence of thermal chromaticity shifts [8].

The main function of a lamp with a programmable spectrum should be the ability to generate light with the required spectrum for each kind of application. Therefore, in order to verify this ability in the developed lamp, a series of significantly different spectral characteristics was generated. In this paper two different spectra, marked $\mathrm{A}$ and $\mathrm{B}$ respectively, were selected for further analysis.

\footnotetext{
*E-mail: m.feldzensztajn@niviss.com
} 
To compare the theoretical and measured spectrum, the correlation function (1) is useful.

$$
\operatorname{Correl}(X, Y)=\frac{\sum(x-\bar{x})(y-\bar{y})}{\sqrt{\sum(x-\bar{x})^{2} \sum(y-\bar{y})^{2}}} .
$$

where $\bar{x}$ and $\bar{y}$ are the sample means average ( $x$ data) and average ( $y$ data).

Additionally, the gamut index and fidelity index as well as colour temperature for the both modelled and measured spectra were calculated.

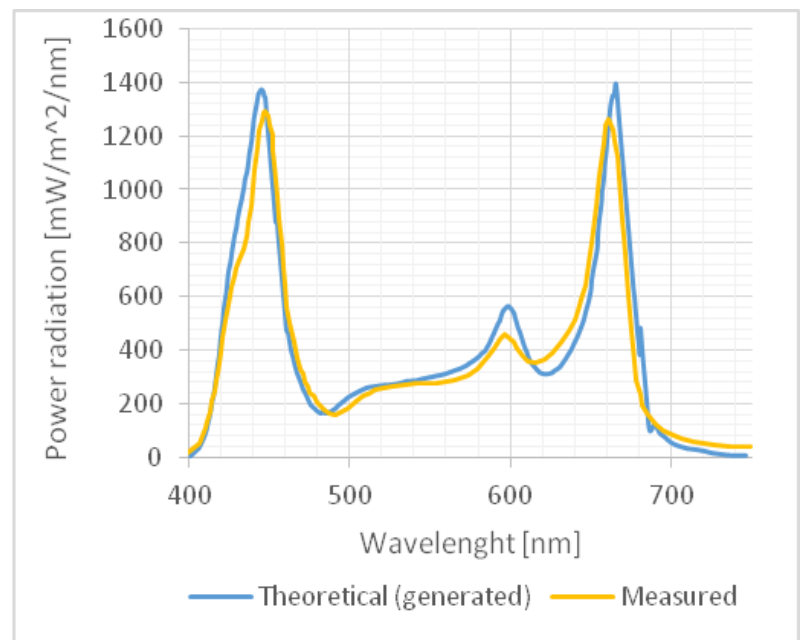

Fig. 2. Comparison of modelled and measured spectrum A.

The spectral characteristics of modelled and measured spectra (spectrum A) are presented in Fig. 2, while the gamut index and fidelity index are presented in $R_{f}-R_{g}$ plot (Fig. 3). Calculated parameters are shown in Table 1.

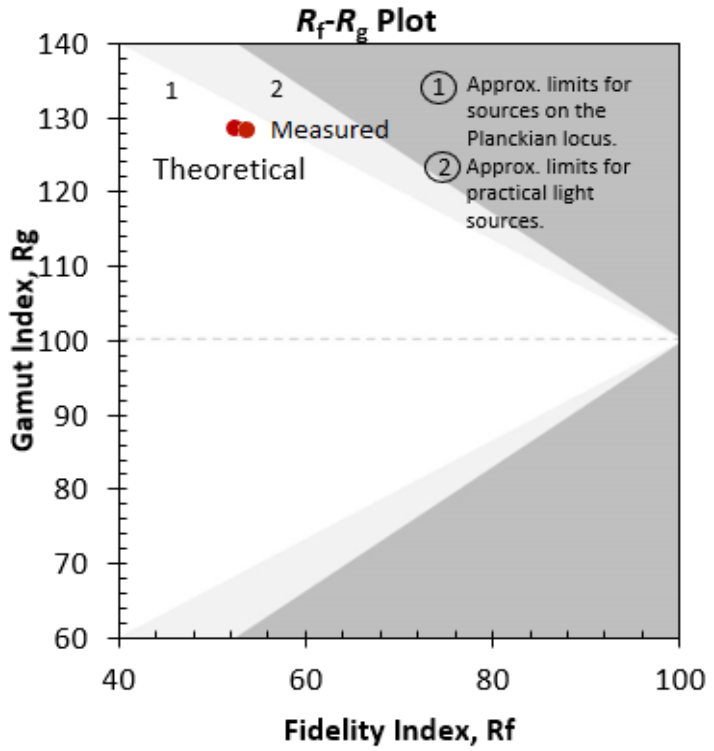

Fig. 3. Differences between measured and modelled values of gamut index and fidelity index (spectrum A).

Table 1. Parameters calculated for Spectrum A

\begin{tabular}{|c|c|c|}
\hline parameter & $\begin{array}{c}\text { Theoretical } \\
\text { spectrum }\end{array}$ & $\begin{array}{c}\text { Measured } \\
\text { spectrum }\end{array}$ \\
\hline$R_{f}$ & 50.6 & 52.1 \\
\hline$R_{g}$ & 129.7 & 128.9 \\
\hline CCT $(\mathrm{K})$ & 8257 & 8978 \\
\hline Correlation & \multicolumn{2}{|c|}{0.921} \\
\hline
\end{tabular}

Obtained parameters are similar for both examined spectra, e.g. the differences of $R_{f}$ and $R_{g}$ are equal to 0.8 and 1.5 , respectively.

Similarly, the spectral characteristics of modelled and measured spectra for spectrum B are presented in Fig. 4, and the gamut index and fidelity index $\left(\mathrm{R}_{\mathrm{f}}-\mathrm{R}_{\mathrm{g}} \mathrm{plot}\right)$ are presented in Fig. 5. Calculated parameters are shown in Table 2. 


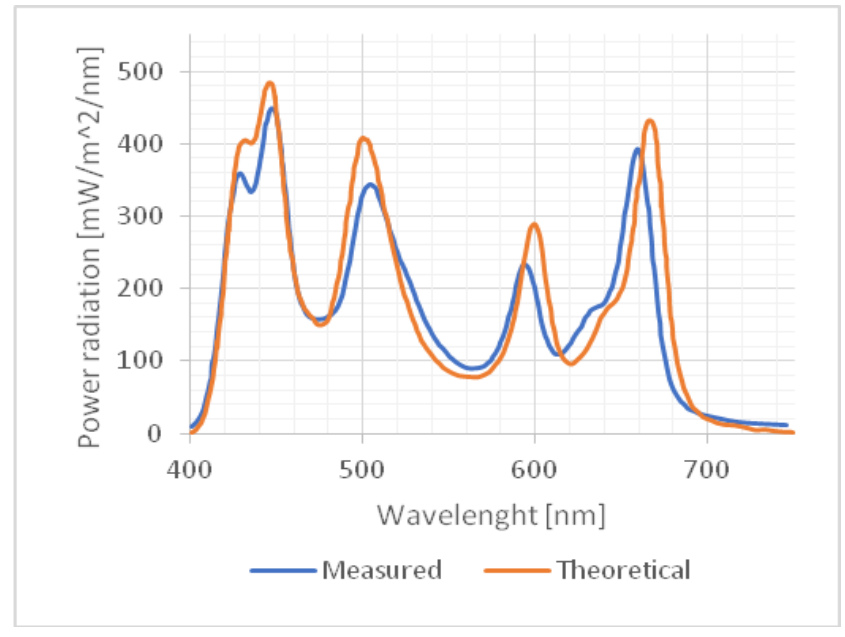

Fig. 4. Comparison of modelled and measured spectrum B.

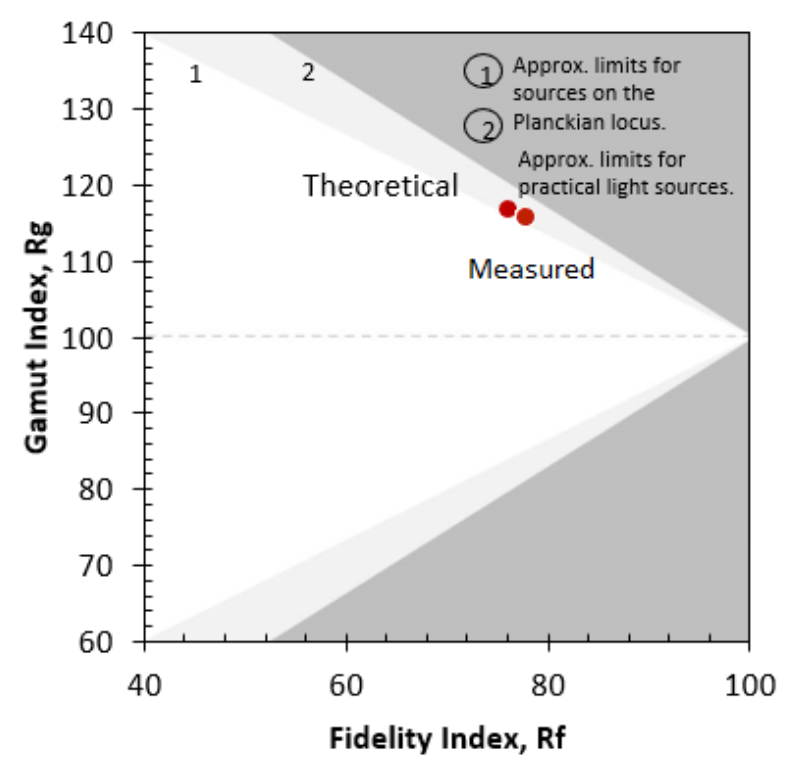

Fig. 5. Differences between measured and modelled values of the gamut index and fidelity index (spectrum B).

Table 2. Parameters calculated for Spectrum B

\begin{tabular}{|l|l|l|}
\hline Parameter & $\begin{array}{l}\text { Theoretical } \\
\text { spectrum }\end{array}$ & $\begin{array}{l}\text { Measured } \\
\text { spectrum }\end{array}$ \\
\hline$R_{f}$ & 75.1 & 77.1 \\
\hline$R_{g}$ & 117.2 & 116 \\
\hline CCT $(\mathrm{K})$ & 9889 & 11701 \\
\hline Correlation & \multicolumn{2}{|c|}{0.87} \\
\hline
\end{tabular}

For Spectrum B, the obtained parameters for calculated and measured spectra differ more than for Spectrum A. However, the differences are still relatively small.

There are some differences between the examined spectra, but they are acceptable for engineering tests and fast prototyping purposes. Differences in the spectrum arise because of non-ideal linear characteristics of optical performance as a function of LED current. Other issue is connected with dimming method. In the presented lamp, dimming is realized by a pulse width modulation (PWM) method and can have a significant influence for the final result [9].

Summarizing, in this Letter, the performance of the lamp with an adjustable spectrum, based on several LEDs driven independently, was assessed. Comparison was made between selected calculated spectra and corresponding spectra of emitted light. Performed measurements show a satisfactory agreement of emitted light spectra with calculated ones. The accuracy of calculations can be further improved by taking into account the shift of spectra with the LED junction temperature.

This study was supported by DS Programs of the Faculty of Electronics, Telecommunications and Informatics of the Gdańsk University of Technology.

\section{References}

[1] P. Mottier, LEDs for Lighting Applications (Willey 2009).

[2] S. Pimputkar, J.S. Speck, S.P. DenBaars, S. Nakamura, Nature Photonics 3(4), 180 (2009).

[3] A.J. Knulst, R. Mooijweer, F.W. Jansen, L.P. Stassen, J. Dankelman, Minim. Invasive Ther. Allied Technol. 20(5), 97 (2011).

[4] U. Blaszczak, L. Gryko, A. Palkowska, E. Kulesza, A. Zajac, Lighting Conference of the Visegrad Countries (Lumen), IEEE (17 November 2016); doi: 10.1109/LUMENV.2016.7745522.

[5] K. Nanya, Y. Ishigami, S. Hikosaka, E. Goto, Acta Hortic. 956 , 264 (2012); doi:10.17660/ActaHortic.2012.956.29.

[6] T. Cegielski, D. Bujalski, K. Kowalczyk, J. Gajc-Wolska, L. Hemka, Proc. Electrotechn. Inst. 63(273), 79 (2016); doi:10.5604/00326216.1210748.

[7] A. Mazikowski, M. Feldzensztajn, Proc. SPIE 10445, 104450K (2017); doi:10.1117/12.2280920.

[8] CREE Inc, Cree ${ }^{\circledR}$ XLamp $®$ XT-E LEDs Product family data sheet 2018, http://www.cree.com/led-components/media/documents/ XLampXTE.pdf (accessed 16 April 2018).

[9] M. Dyble, N. Narendran, A. Bierman, T. Klein, Optics and Photonics Conference 2005, San Diego, California, United States. 